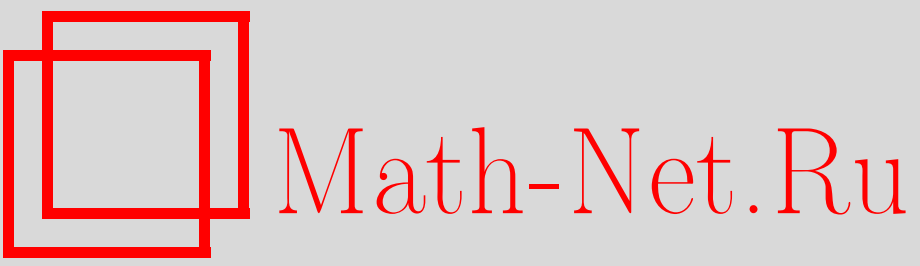

В. В. Андреев, Процессы реакция-диффузия и «мертвая зона» в пористой грануле катализатора, Итоги науки и техн. Сер. Соврем. мат. и ее прил. Темат. обз., 2020, том 186, 3-12

DOI: https://doi.org/10.36535/0233-6723-2020-186-3-12

Использование Общероссийского математического портала Math-Net.Ru подразумевает, что вы прочитали и согласны с пользовательским соглашением

http://www.mathnet.ru/rus/agreement

Параметры загрузки:

IP : 52.205 .19 .152

26 апреля 2023 г., 18:34:33 


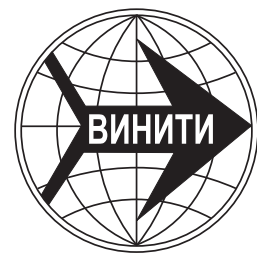

ИТОГИ НАУКИ И ТЕХНИКИ.

Современная математика и ее приложения.

Тематические обзоры.

Том 186 (2020). С. 3-12

DOI: $10.36535 / 0233-6723-2020-186-3-12$

УДК 517.928; 544.47

\title{
ПРОЦЕССЫ РЕАКЦИЯ-ДИФФУЗИЯ И «МЕРТВАЯ ЗОНА» В ПОРИСТОЙ ГРАНУЛЕ КАТАЛИЗАТОРА
}

\author{
(c) 2020 г. $\quad$ В. В. АНДРЕЕВ
}

\begin{abstract}
АннотАция. Исследованы реакционно-диффузионные процессы в пористых гранулах катализатора шарообразной, цилиндрической и пластинчатой форм для различных типов химических реакций, протекающих в них. Получены приближенные решения квазигомогенной модели для каталитических реакций общего вида. Проанализированы необходимые условия возникновения «мертвой зоны» в центральной области пористой гранулы катализатора в случае протекания каталитической реакции общего вида.
\end{abstract}

Ключевъе слова: гетерогенный катализ, реакционно-диффузионный процесс, пористая гранула катализатора, «мертвая зона».

\section{REACTION-DIFFUSION PROCESSES AND “DEAD ZONE" IN A POROUS CATALYST GRANULE}

\author{
(c) 2020 V. V. ANDREEV
}

\begin{abstract}
In this paper, we discuss reaction-diffusion processes in porous catalyst granules of spherical, cylindrical, and lamellar forms for various types of chemical reactions. We obtain approximate solutions of the quasi-homogeneous model for general catalytic reactions. Also, we analyze necessary conditions for the appearance of a "dead zone" in the central domain of a porous catalyst granule in the case of a general catalytic reaction.
\end{abstract}

Keywords and phrases: heterogeneous catalysis, reaction-diffusion process, porous catalyst granule, "dead zone".

AMS Subject Classification: 34C60, 35K05, 35K57

1. Введение. В гетерогенном катализе, как правило, молекулы катализатора наносятся на поверхность твердых гранул, а исходные вещества, участвующие в каталитических превращениях, поступают в жидком или газообразном состоянии. Размер гранул составляет порядка 1 см. С целью увеличения площади активной поверхности гранулы выполняются в виде высокопористых структур. В этом случае удельная площадь активной поверхности пористой гранулы катализатора достигает сотен м² на 1 грамм вещества гранулы. Однако из-за диффузионного торможения в порах концентрации исходных реагентов в глубине гранулы ниже, чем у ее внешней поверхности (т. е. концентрации исходных реагентов падают по направлению к центральной области гранулы). Чем выше скорость гетерогенной каталитической реакции и сильнее диффузионное торможение в порах, тем значительнее падение концентраций исходных реагентов по глубине гранулы. В некоторых случаях уменьшение концентраций по глубине пористой гранулы может быть настолько большим, что реагенты совсем не поступают в центральную область гранулы. Такую область называют «мертвой зоной». Следовательно, эффективность каталитического процесса 
снижается в центральных областях пористой гранулы. Таким образом, важны исследования, направленные на повышение эффективности гетерогенных каталитических процессов в пористых гранулах. В таких исследованиях рассматриваются различные аспекты достижения большей эффективности пористого катализатора. Так, в работах $[1,17]$ исследована возможность повышения эффективности такого каталитического процесса путем неоднородного размещения катализатора по глубине пористой гранулы. Возможность достижения большей эффективности пористых катализаторов путем размещения в центральной области гранулы непроницаемого ядра исследована в работе [4]. Другим способом повышения эффективности пористых гранул катализатора является осуществление каталитической реакции в них в искусственно создаваемом нестационарном режиме $[15,16,18]$. В работе [14] получены условия максимизации производительности пористой гранулы катализатора с управляемым профилем активности при осуществлении каталитического процесса в ней в искусственно создаваемом нестационарном режиме. Необходимые условия возникновения «мертвой зоны» для произвольных каталитических реакций в пористых гранулах катализатора сферической, цилиндрической и пластинчатой форм получены и исследованы, в частности, в работе [3].

2. Математическая модель. Рассмотрим химические реакции, протекающие на внутренней активной поверхности пористой гранулы катализатора:

$$
0=\sum_{i=1}^{n} a_{i}^{p} A_{i}, \quad p=1, \ldots, k .
$$

Здесь $a_{i}^{p}$-стехиометрические коэффициенты $\left(a_{i}^{p}<0\right.$ для исходных веществ, а для продуктов реакций $\left.a_{i}^{p}>0\right) ; A_{i}$ - символы участвующих в реакциях (1) веществ; номер (маршрут) реакции в схеме (1) обозначен через $p$. Скорость реакции, протекающей по маршруту $p$ и отнесенной к единице объема пористой гранулы катализатора, обозначим через $r_{p}=r_{p}(x)=r_{p}\left(C_{1}, \ldots, C_{n}, T\right)$. Концентрация вещества $A_{i}$ обозначена через $C_{i}$, а температура $-T$. Ввиду того, что $C_{i}$ и $T$ в пористой грануле катализатора являются функциями пространственных координат, а также времени $t$ в случае нестационарного режима каталитического процесса, функции $r_{p}$ являются сложными функциями этих независимых переменных.

В случае каталитических реакций, протекающих в соответствии со схемой (1) в пористой грануле катализатора пластинчатой, цилиндрической или шарообразной форм, уравнения баланса масс реагентов и продуктов реакций, а также тепла имеют вид [3]:

$$
\begin{gathered}
\frac{\partial\left(b C_{i}\right)}{\partial t}-x^{-\alpha} \frac{\partial}{\partial x}\left(x^{\alpha} D_{i}^{*} \frac{\partial C_{i}}{\partial x}\right)-\sum_{p=1}^{k} g a_{i}^{p} r_{p}=0, \quad i=1, \ldots, n \\
\frac{\partial(c \rho T)}{\partial t}-x^{-\alpha} \frac{\partial}{\partial x}\left(x^{\alpha} \lambda^{*} \frac{\partial T}{\partial x}\right)-\sum_{p=1}^{k} g\left(-\Delta H_{p}\right) r_{p}=0 .
\end{gathered}
$$

Здесь предполагается, что диффузия веществ $A_{i}$ в порах является независимой от присутствия остальных и подчиняется закону Фика, а теплопроводность гранулы - закону Фурье. В уравнениях (2) и (3) использованы следующие обозначения: $x$ - текущая пространственная координата; $b$ - коэффициент пористости, который в общем случае является функцией пространственных координат и времени; $\alpha$ - параметр, определяющийся геометрической формой гранулы $(\alpha=2-$ шарообразная, $\alpha=1$-цилиндрическая, $\alpha=0$ - пластинчатая); $D_{i}^{*}$ - эффективный коэффициент диффузии вещества $A_{i}$ в порах гранулы катализатора; $\lambda^{*}$ - эффективный коэффициент теплопроводности гранулы катализатора; $g=g(x, t)$ - плотность распределения молекул катализатора в грануле, которая в общем случае является функцией пространственных координат и времени; $\left(-\Delta H_{p}\right)$ - энтальпия реакции в схеме $(1)$, протекающей по маршруту $p ; c$ и $\rho-$ соответственно эффективные удельная теплоемкость и плотность системы «пористая гранула катализатора - реакционная смесь, заполнившая поры».

Система дифференциальных уравнений параболического типа (2), (3) представляет собой так называемую квазигомогенную модель реакционно-диффузионного процесса в пористой грануле 
катализатора. Ее суть состоит в том, что пористые гранулы катализатора рассматривают как гомогенную среду, в которой протекает каталитическая реакция с некоторой скоростью, а перенос веществ в порах характеризуется эффективными коэффициентами диффузии. Такой подход оправдан тем, что отдельные образования пористой структуры имеют размеры от десятков до десятков тысяч ангстрем, а величина гранулы - порядка нескольких миллиметров и одна пористая гранула катализатора содержит от $10^{9}$ до $10^{14}$ (или более) мелких частиц. Вследствие этого для описания процессов в пористой грануле катализатора можно применить общие статистические подходы. Необходимо отметить, что многочисленные экспериментальные данные, приведенные, в частности, в работе [23], свидетельствуют о том, что реакционно-диффузионные процессы в пористых гранулах катализатора хорошо описываются квазигомогенной моделью.

В силу наличия у рассматриваемых гранул сферической, цилиндрической и осевой симметрий справедливы следующие граничные условия при $x=0$ :

$$
\begin{aligned}
\left.D_{i}^{*} \frac{\partial C_{i}}{\partial x}\right|_{x=0} & =0, \quad i=1, \ldots, n ; \\
\left.\lambda^{*} \frac{\partial T}{\partial x}\right|_{x=0} & =0 .
\end{aligned}
$$

На внешней поверхности пористой гранулы катализатора при $x=R_{S}$ граничные условия, выражающие непрерывность потоков веществ $A_{i}$ и потока тепла в пограничном слое вокруг гранулы, имеют соответственно вид:

$$
\begin{aligned}
\left.D_{i}^{*} \frac{\partial C_{i}}{\partial x}\right|_{x=R_{S}} & =\beta_{i}\left(C_{0 i}-C_{S i}\right), \quad i=1, \ldots, n \\
\left.\lambda^{*} \frac{\partial T}{\partial x}\right|_{x=0} & =\beta_{T}\left(T_{0}-T_{S}\right) .
\end{aligned}
$$

Здесь $C_{S i}$ - концентрация вещества $A_{i}$ около внешней поверхности пористой гранулы; $T_{S}-$ температура на внешней поверхности пористой гранулы; $C_{0 i}$ и $T_{0}$ - соответственно концентрация вещества $A_{i}$ и температура на таком расстоянии от внешней поверхности гранулы, на котором можно пренебречь влиянием последней на свойства и режим течения внешней реакционной смеси; $\beta_{i}$ - коэффициент массообмена через пограничный слой по веществу $A_{i}$ между внешней поверхностью гранулы и окружающей ее реакционной смесью; $\beta_{T}$ - коэффициент теплообмена через пограничный слой между внешней поверхностью гранулы и окружающей ее реакционной смесью.

Начальные условия для системы дифференциальных уравнений (2) и (3) зададим в виде:

$$
\begin{aligned}
C_{i}\left(x, t_{0}\right) & =C_{\infty i}(x), \quad i=1, \ldots, n ; \\
T\left(x, t_{0}\right) & =T_{\infty}(x) .
\end{aligned}
$$

Наблюдаемая скорость расходования (образования) вещества $A_{i}$ в реакциях $(1)$, протекающих в пористых гранулах катализатора сферической, цилиндрической и пластинчатой форм, равна:

$$
J_{i}=-\left.S_{0} D_{i}^{*} \frac{\partial C_{i}}{\partial x}\right|_{x=R_{S}}, \quad i=1, \ldots, n .
$$

Здесь $S_{0}$ - площадь внешней поверхности гранулы катализатора, равная $S_{0}=4 \pi R_{S}^{2}$ в случае сферической гранулы, $S_{0}=2 \pi R_{S} l$ в случае цилиндрической гранулы $\left(l-\right.$ высота цилиндра), $S_{0}=2 S_{x}$ в случае гранулы пластинчатой формы $\left(S_{x}\right.$ - площадь поверхности пластины, перпендикулярной оси $x$ ). Поверхность $S_{0}$ проницаема для веществ $A_{i}$. Эффективность пористой гранулы катализатора при осуществлении в ней каталитических реакций вида (1) определяется величиной $\eta_{i}$, называемой фактором эффективности по веществу $A_{i}$, и вычисляется по формуле

$$
\eta_{i}=\frac{J_{i}}{J_{0 i}}, \quad J_{0 i}=g_{0} V_{0} \sum_{p=1}^{k} a_{i}^{p} r_{0 p} .
$$


Здесь $V_{0}$ - объем пористой гранулы катализатора, равный $V_{0}=4 \pi R_{S}^{3} / 3$ в случае сферической гранулы, $V_{0}=\pi R_{S}^{2} l$ в случае цилиндрической гранулы, $V_{0}=2 S_{x} R_{S}$ в случае гранулы пластинчатой формы; $r_{0 p}=r_{p}\left(C_{01}, \ldots, C_{0 n}, T_{0}\right) ; J_{0 i}$ - наблюдаемая скорость расходования (образования) вещества $A_{i}$ в реакциях (1) в предположении, что концентрации всех веществ, а также температура неизменны по глубине пористой гранулы катализатора, а молекулы катализатора распределены равномерно по глубине, т. е. $g(x, t)=g_{0} \equiv$ const. Кроме того, предполагается отсутствие пограничного слоя у внешней поверхности гранулы, затрудняющего массо- и теплообмен между ней и обтекающим ее потоком внешней реакционной смеси. Отношение величин $S_{0}$ и $V_{0}$ равно $S_{0} / V_{0}=(\alpha+1) / R_{S}$. Тогда с учетом формул (10) и (11) для вычисления величины $\eta_{i}$ справедлива формула

$$
\eta_{i}=-\left.\frac{(\alpha+1) D_{i}^{*}}{g_{0} R_{S} \sum_{p=1}^{k} a_{i}^{p} r_{0 p}} \frac{\partial C_{i}}{\partial x}\right|_{x=R_{S}} .
$$

3. Приближенное решение математической модели. В работе [6] был предложен алгоритм нахождения приближенного решения математической модели (2) и (3) с граничными условиями (4)-(7) для реакции вида (1), протекающей в стационарном режиме, т. е. при

$$
\frac{\partial\left(b C_{i}\right)}{\partial t} \equiv 0, \quad i=1, \ldots, n ; \quad \frac{\partial(c \rho T)}{\partial t} \equiv 0 .
$$

Используя уравнения (6), (7) и (12) выразим концентрации веществ и температуры на внешней поверхности гранулы через их величины в ядре внешнего потока реакционной смеси:

$$
C_{S i}=C_{0 i}-U_{i}, \quad i=1, \ldots, n+1 ; \quad U_{i}=-\frac{\eta_{i} g_{0} R_{S}^{2}}{(\alpha+1) D_{i}^{*} B_{i}} \sum_{p=1}^{k} a_{i}^{p} r_{0 p} .
$$

Здесь $C_{S n+1}=T_{S} ; C_{0 n+1}=T_{0} ; D_{n+1}^{*}=\lambda^{*} ; a_{n+1}^{p}=-\Delta H_{p} ; B_{i}=\beta_{i} R_{S} / D_{i}^{*}$ - безразмерный параметр, характеризующий сопротивление массо- и теплообмену в пограничном слое; $\beta_{n+1}=\beta_{T}$. При выводе приближенного решения квазигомогенной модели $(2),(3)$ в стационарном режиме (т. е. выполняются равенства (13)) рассматривается случай равномерного распределения молекул катализатора по глубине пористой гранулы $g(x, t)=g_{0} \equiv$ const.

Функцию $r_{s p}=r_{p}\left(C_{S 1}, \ldots, C_{S n}, T_{S}\right)$, описывающую скорость каталитической реакции по маршруту $p$ в схеме (1) на внешней поверхности пористой гранулы катализатора, разложим в ряд Тейлора по степеням $U_{i}, i=1, \ldots, n+1$. Тогда, воспользовавшись выражениями (14), получим

$$
\begin{aligned}
r_{s p}=r_{0 p}-\frac{g_{0} R_{S}^{2}}{\alpha+1} \sum_{l=1}^{k} & \left.\sum_{i=1}^{n+1} r_{0 l} \frac{a_{i}^{l} \eta_{i}}{D_{i}^{*} B_{i}} \frac{\partial r_{s p}}{\partial U_{i}}\right|_{U_{1}=0, \ldots, U_{n+1}=0}+ \\
& +\left.\frac{g_{0}^{2} R_{S}^{4}}{2(\alpha+1)^{2}} \sum_{l=1}^{k} \sum_{f=1}^{k} \sum_{i=1}^{n+1} \sum_{j=1}^{n+1} r_{0 l} r_{0 f} \frac{a_{i}^{l} a_{j}^{f} \eta_{i} \eta_{j}}{D_{i}^{*} D_{j}^{*} B_{i} B_{j}} \frac{\partial^{2} r_{s p}}{\partial U_{i} \partial U_{j}}\right|_{U_{1}=0, \ldots, U_{n+1}=0}+\ldots
\end{aligned}
$$

Предположим, что $D_{i}^{*} \equiv \mathrm{const}, i=1, \ldots, n+1 ; \Delta H_{p} \equiv \mathrm{const}, p=1, \ldots, k$. Для нахождения коэффициентов искомого ряда, являющегося приближенным решением квазигомогенной модели (2), (3) в стационарном режиме (т. е. справедливы равенства (13)), с помощью уравнений (2) и (3) вычислим производные:

$$
\begin{aligned}
& \frac{d^{2} C_{i}}{d x^{2}}=-\frac{\alpha}{x} \frac{d C_{i}}{d x}-\frac{g_{0}}{D_{i}^{*}} \sum_{p=1}^{k} a_{i}^{p} r_{p}, \quad i=1, \ldots, n+1 ; \\
& \frac{d^{3} C_{i}}{d x^{3}}=-\frac{\alpha}{x} \frac{d^{2} C_{i}}{d x^{2}}+\frac{\alpha}{x^{2}} \frac{d C_{i}}{d x}-\frac{g_{0}}{D_{i}^{*}} \sum_{p=1}^{k} a_{i}^{p} \frac{d r_{p}}{d x}, \quad i=1, \ldots, n+1 ; \\
& \frac{d^{4} C_{i}}{d x^{4}}=-\frac{\alpha}{x} \frac{d^{3} C_{i}}{d x^{3}}+\frac{2 \alpha}{x^{2}} \frac{d^{2} C_{i}}{d x^{2}}-\frac{2 \alpha}{x^{3}} \frac{d C_{i}}{d x}-\frac{g_{0}}{D_{i}^{*}} \sum_{p=1}^{k} a_{i}^{p} \frac{d^{2} r_{p}}{d x^{2}}, \quad i=1, \ldots, n+1 .
\end{aligned}
$$


Используя правило дифференцирования сложных функций и раскрывая неопределенности в выражениях (16)-(18) по правилу Лопиталя при $x=0$ получим $(i=1, \ldots, n+1 ; p=1, \ldots, k)$ :

$$
\begin{aligned}
& \left.\frac{d C_{i}(x)}{d x}\right|_{x=0}=0 ; \\
& \left.\frac{d r_{p}(x)}{d x}\right|_{x=0}=0 ; \\
& \left.\frac{d^{2} C_{i}(x)}{d x^{2}}\right|_{x=0}=-\frac{g_{0}}{(\alpha+1) D_{i}^{*}} \sum_{l=1}^{k} a_{i}^{l} r_{l}(0) \\
& \left.\frac{d^{2} r_{p}(x)}{d x^{2}}\right|_{x=0}=-\left.\frac{g_{0}}{\alpha+1} \sum_{l=1}^{k} \sum_{j=1}^{n+1} \frac{a_{j}^{l} r_{l}(0)}{D_{j}^{*}} \frac{\partial r_{p}(x)}{\partial C_{j}}\right|_{x=0} \\
& \left.\frac{d C_{i}^{3}(x)}{d x^{3}}\right|_{x=0}=0 ; \\
& \left.\frac{d r_{p}^{3}(x)}{d x^{3}}\right|_{x=0}=0 ; \\
& \left.\frac{d C_{i}^{4}(x)}{d x^{4}}\right|_{x=0}=\left.\frac{3 g_{0}^{2}}{(\alpha+1)(\alpha+3)} \sum_{t=1}^{k} \sum_{l=1}^{k} \sum_{j=1}^{n+1} \frac{a_{i}^{t} a_{j}^{l} r_{l}(0)}{D_{i}^{*} D_{j}^{*}} \frac{\partial r_{t}(x)}{\partial C_{j}}\right|_{x=0} ; \\
& \left.\frac{d r_{p}^{4}(x)}{d x^{4}}\right|_{x=0}=\left.\frac{3 g_{0}^{2}}{(\alpha+1)^{2}} \sum_{t=1}^{k} \sum_{l=1}^{k} \sum_{m=1}^{n+1} \sum_{j=1}^{n+1} \frac{a_{m}^{t} a_{j}^{l} r_{t}(0) r_{l}(0)}{D_{m}^{*} D_{j}^{*}} \frac{\partial^{2} r_{p}(x)}{\partial C_{m} \partial C_{j}}\right|_{x=0}+ \\
& +\left.\left.\frac{3 g_{0}^{2}}{(\alpha+1)(\alpha+3)} \sum_{t=1}^{k} \sum_{l=1}^{k} \sum_{m=1}^{n+1} \sum_{j=1}^{n+1} \frac{a_{m}^{t} a_{j}^{l} r_{l}(0)}{D_{m}^{*} D_{j}^{*}} \frac{\partial r_{t}(x)}{\partial C_{j}}\right|_{x=0} \frac{\partial r_{p}(x)}{\partial C_{m}}\right|_{x=0} .
\end{aligned}
$$

Здесь запись $r_{t}(0)$ означает, что функции $r_{t}(x)(t=1, \ldots, k)$ вычисляются в точке $x=0$. Таким образом, функция $r_{p}(x)$ с учетом выражений $(20)$ и $(24)$ представляется в виде следующего ряда

$$
r_{p}(x)=r_{p}(0)+\left.\frac{x^{2}}{2} \frac{d r_{p}^{2}(x)}{d x^{2}}\right|_{x=0}+\left.\frac{x^{4}}{24} \frac{d r_{p}^{4}(x)}{d x^{4}}\right|_{x=0}+\ldots
$$

Для определения фактора эффективности будет удобнее пользоваться не соотношением (12), а другим, равносильным ему, выражением. За единицу времени в пористой грануле катализатора объемом $V_{0}$ образуется (расходуется) количество вещества $A_{i}$, равное

$$
\int_{V_{0}} g \sum_{p=1}^{k} a_{i}^{p} r_{p} d V
$$

В последней формуле $g$ является в общем случае функцией всех трех пространственных координат. Тогда для определения фактора эффективности $\eta_{i}$ получим

$$
\eta_{i}=(\alpha+1) \int_{0}^{R_{S}} g(x) \sum_{p=1}^{k} a_{i}^{p} r_{p}(x) x^{\alpha} d x /\left(g_{0} \sum_{p=1}^{k} a_{i}^{p} r_{0 p}\right) .
$$

В формулы (22) и (26) входят частные производные

$$
\left.\frac{\partial r_{p}(x)}{\partial C_{j}}\right|_{x=0}
$$


от которых необходимо перейти к

$$
\left.\frac{\partial r_{p}(x)}{\partial C_{j}}\right|_{x=R_{S}}
$$

Для этого запишем ряд Тейлора:

$$
\left.\frac{\partial r_{p}(x)}{\partial C_{j}}\right|_{x=R_{S}}=\left.\frac{\partial r_{p}(x)}{\partial C_{j}}\right|_{x=0}+\left.\frac{d}{d x}\left(\frac{\partial r_{p}(x)}{\partial C_{j}}\right)\right|_{x=0}+\left.\frac{1}{2} \frac{d^{2}}{d x^{2}}\left(\frac{\partial r_{p}(x)}{\partial C_{j}}\right)\right|_{x=0}+\ldots
$$

Воспользовавшись равенствами (19) и (21), преобразуем формулу (29) к виду

$$
\left.\frac{\partial r_{p}(x)}{\partial C_{j}}\right|_{x=0}=\left.\frac{\partial r_{p}(x)}{\partial C_{j}}\right|_{x=R_{S}}+\left.\frac{g_{0}}{2(\alpha+1)} \sum_{t=1}^{k} \sum_{l=1}^{n+1} \frac{a_{l}^{t} r_{t}(0)}{D_{l}^{*}} \frac{\partial^{2} r_{p}}{\partial C_{j} \partial C_{l}}\right|_{x=0}+\ldots
$$

В формулах (26) и (30) заменим

на

$$
\left.\frac{\partial^{2} r_{p}}{\partial C_{j} \partial C_{l}}\right|_{x=0}
$$

$$
\left.\frac{\partial^{2} r_{p}}{\partial C_{j} \partial C_{l}}\right|_{x=R_{S}}
$$

так как, если ограничиться первыми тремя членами ряда (27), такая замена сказывается только на членах ряда, содержащих $x$ в степени большей 4. Подставим ряд (27) в выражение (28) и вычислим интеграл. Затем с помощью формул $(14),(15)$ и $(27)$ в полученном выражении перейдем от величин $r_{p}(0)$ к $r_{0 p}, p=1, \ldots, k$. В результате приходим к следующему выражению для приближенного определения фактора эффективности пористой гранулы катализатора по веществу $A_{m}, m=1, \ldots, n$ :

$$
\begin{aligned}
\eta_{m}=1+ & \left(\sum_{p=1}^{k} a_{m}^{p} r_{0 p}\right)^{-1}\left[\frac{g_{0} R_{S}^{2}}{(\alpha+1)(\alpha+3)}\left\{\sum_{f=1}^{k} \sum_{t=1}^{k} a_{m}^{f} r_{0 t} \Delta_{t f}+(\alpha+3) \sum_{l=1}^{n+1} \frac{\Delta^{m l}}{D_{l}^{*} B_{l}}\right\}+\right. \\
& +\frac{g_{0}^{2} R_{S}^{4}}{(\alpha+1)^{2}(\alpha+3)(\alpha+5)}\left\{\sum_{f=1}^{k} \sum_{t=1}^{k} \sum_{q=1}^{k} a_{m}^{f} r_{0 t}\left(2 \Delta_{t q} \Delta_{q f}+r_{0 q} \Delta_{t q f}\right)+\right. \\
& +(\alpha+5)\left(\sum_{l=1}^{n+1} \sum_{f=1}^{k} \sum_{t=1}^{k} \frac{a_{l}^{f} r_{0 t} \Delta_{t f} \Delta^{m l}}{D_{l}^{*} B_{l}}\left(\sum_{p=1}^{k} a_{l}^{p} r_{0 p}\right)^{-1}+\sum_{l=1}^{n+1} \sum_{j=1}^{n+1} \frac{\Delta^{m l j}+\Delta_{m}^{l j}}{D_{l}^{*} D_{j}^{*} B_{j}}\right)+ \\
& \left.\left.+\frac{(\alpha+3)(\alpha+5)}{2} \sum_{l=1}^{n+1} \sum_{j=1}^{n+1} \frac{1}{D_{l}^{*} D_{j}^{*} B_{l} B_{j}}\left(\Delta^{m l j}+2 \Delta^{m l} \Delta^{l j}\left(\sum_{p=1}^{k} a_{l}^{p} r_{0 p}\right)^{-1}\right)\right\}+\ldots\right]
\end{aligned}
$$

Введены в формуле (31) следующие обозначения:

$$
\begin{gathered}
\Delta_{\beta q}=-\left.\sum_{i=1}^{n+1} \frac{a_{i}^{\beta}}{D_{i}^{*}} \frac{\partial r_{s q}}{\partial U_{i}}\right|_{U_{1}=0, \ldots, U_{n+1}=0} ; \quad \Delta_{p t f}=\left.\sum_{i=1}^{n+1} \sum_{j=1}^{n+1} \frac{a_{i}^{p} a_{j}^{t}}{D_{i}^{*} D_{j}^{*}} \frac{\partial^{2} r_{s f}}{\partial U_{i} \partial U_{j}}\right|_{U_{1}=0, \ldots, U_{n+1}=0} ; \\
\Delta^{i l}=-\left.\sum_{f=1}^{k} \sum_{t=1}^{k} a_{i}^{f} a_{l}^{t} r_{0 t} \frac{\partial r_{s f}}{\partial U_{l}}\right|_{U_{1}=0, \ldots, U_{n+1}=0} ; \\
\Delta^{i l j}=\left.\sum_{f=1}^{k} \sum_{p=1}^{k} \sum_{t=1}^{k} a_{i}^{f} a_{l}^{p} a_{j}^{t} r_{0 p} r_{0 t} \frac{\partial^{2} r_{s f}}{\partial U_{l} \partial U_{j}}\right|_{U_{1}=0, \ldots, U_{n+1}=0} ; \\
\Delta_{i}^{l j}=\left.\left.\sum_{f=1}^{k} \sum_{p=1}^{k} \sum_{t=1}^{k} a_{i}^{f} a_{j}^{p} a_{l}^{t} r_{0 p} \frac{\partial r_{s f}}{\partial U_{l}}\right|_{U_{1}=0, \ldots, U_{n+1}=0} \frac{\partial r_{s t}}{\partial U_{j}}\right|_{U_{1}=0, \ldots, U_{n+1}=0} ;
\end{gathered}
$$


Приближенные формулы (31) для оценки фактора эффективности пористых гранул катализаторов для произвольных химических реакций (1) представляют интерес в силу того, что модели типа реакция диффузия допускают точные решения только для частных случаев, некоторые из которых исследованы в работах $[19,20,24]$. С помощью приближенных решений математической модели часто удается намного быстрее по сравнению с численными методами анализа оценить, в частности, влияние всех основных параметров, характеризующих процесс, на конечные результаты исследования. Кроме того, они полезны при тестирования численных алгоритмов.

Применим полученную формулу (31) для анализа эффективности пористой гранулы катализатора в случае простой единственной каталитической реакции

$$
A_{1} \Rightarrow \text { Reaction Products. }
$$

Пусть скорость реакции описывается уравнением

$$
r_{1}=k_{01} C_{1}^{n} \exp \left(\frac{E_{1}}{R T}\right) .
$$

Здесь $n$-порядок реакции; $k_{01}$-предэкспоненциальный множитель; $E_{1}$ - энергия активации; $R-$ универсальная газовая постоянная.

При учете членов до второго порядка по степеням $R_{S}$ ряд (31) для приближенного вычисления фактора эффективности для каталитической реакции (32) с кинетикой (33) примет вид:

$$
\eta_{1} \approx 1-\frac{\varphi^{2}}{(\alpha+1)(\alpha+3)}\left\{n\left(1+\frac{\alpha+3}{B_{1}}\right)-\beta \gamma_{1}\left(1+\frac{\alpha+3}{B_{T}}\right)\right\} .
$$

Здесь $\varphi$ - параметр Тиле, определяемый по формуле

$$
\varphi=R_{S} \sqrt{\frac{g_{0} r_{01}}{D_{1}^{*} C_{01}}} .
$$

В формуле (34) использованы еще следующие безразмерные величины:

$$
\gamma_{1}=\frac{E_{1}}{R T_{0}} ; \quad \beta=\frac{D_{1}^{*} C_{01}(-\Delta H)}{\lambda^{*} T_{0}} .
$$

Таким образом, формула (34) содержит все параметры, характеризующие каталитическую реакцию (32) с кинетикой (33), протекающую в пористых гранулах катализатора. Ввиду простоты выражения (32) с его помощью легко оценить зависимость фактора эффективности от различных величин, определяющих каталитический процесс (32) с кинетикой (33) в пористых гранулах катализатора.

4. Исследование «мертвой зоны» в пористой грануле катализатора. При осуществлении химических реакций в пористых гранулах катализатора вследствие диффузионного торможения в порах происходит падение концентраций исходных веществ по направлению к центру. При этом стремятся к созданию таких условий, когда концентрации исходных веществ в центре гранулы составляют по крайней мере не менее $70 \%$ от их величин у внешней поверхности гранулы. Поэтому важно знать условия, с помощью которых можно оценивать интенсивность падения концентраций исходных веществ в порах по направлению к центру. Так, в случае сильного диффузионного торможения в порах в центральной области пористой гранулы может возникнуть так называемая «мертвая зона», в которую одно или несколько исходных веществ совсем не поступают. Различные аспекты проявления «мертвой зоны» исследованы, в частности, в работах $[2,5,7-9,12,13,26]$. Необходимые условия возникновения «мертвой зоны» для произвольных каталитических реакций вида (1) исследованы в работах $[3,10,11]$. Для квазигомогенной модели (2), (3) в случае, если скорости реакций $r_{p},(p=1, \ldots, k)$ непрерывны по температуре $T$, а энтальпии реакций $\left(-\Delta H_{p}\right),(p=1, \ldots, k)$ являются непрерывными функциями всех своих аргументов, то необходимые условия возникновения «мертвой зоны» по веществу $A_{j}$ записываются 
в виде:

$$
\begin{gathered}
\lim _{C_{1} \rightarrow C_{1 j}, \ldots, C_{n} \rightarrow C_{n j}, T \rightarrow T_{j}}\left[C_{j} \sum_{p=1}^{k} g(x) a_{j}^{p} r_{p}\left(C_{1}, \ldots, C_{n}, T\right)\right]^{-1}=-\infty ; \\
\lim _{C_{1} \rightarrow C_{1 j}, \ldots, C_{n} \rightarrow C_{n j}, T \rightarrow T_{j}} \frac{\sum_{p=1}^{k} g(x) a_{j}^{p} r_{p}\left(C_{1}, \ldots, C_{n}, T\right)}{D_{j}^{*} C_{j}}=-\infty .
\end{gathered}
$$

Здесь $C_{i j}=C_{i}\left(x_{0 j}\right), i=1, \ldots, n ; T_{j}=T\left(x_{0 j}\right)$. Величина $x_{0 j}$ представляет собой размер «мертвой зоны» по веществу $A_{j}$. Следовательно, в центральной области пористой гранулы $0 \leqslant x \leqslant x_{0 j}$ концентрация вещества $A_{j}$ тождественно равна нулю, т. е. $C_{j}(x) \equiv 0$. Таким образом, в формулах $(35)$ и (36) для вещества, по которой проверяется выполнение необходимых условий возникновения «мертвой зоны», имеем $C_{j j}=C_{j}\left(x_{0 j}\right)=0$.

Применим необходимые условия возникновения «мертвой зоны» (35) и (36) к простой единственной каталитической реакции (32) с кинетикой (33) с учетом того, что в этом случае $p=1$ и, в соответствии со схемой $(1), a_{1}^{1}=-1$ :

$$
\begin{gathered}
\lim _{C_{1} \rightarrow 0, T \rightarrow T_{1}}\left[g(x) k_{01} C_{1}^{n+1} \exp \left(\frac{E_{1}}{R T}\right)\right]^{-1}=\infty ; \\
\lim _{C_{1} \rightarrow 0, T \rightarrow T_{1}} \frac{g(x) k_{01} C_{1}^{n-1}}{D_{1}^{*}} \exp \left(\frac{E_{1}}{R T}\right)=\infty .
\end{gathered}
$$

Таким образом, для всех областей в пористой грануле катализатора, в которых эффективный коэффициент диффузии $D_{1}^{*}$ вещества $A_{1}$ и плотность распределения молекул катализатора $g(x)$ не зависят от концентрации $C_{1}$, из формул (37) и (38) вытекает следующее необходимое условие возникновения «мертвой зоны»:

$$
-1<n<1
$$

При необходимости выяснить возможность одновременного появления «мертвой зоны» по двум веществам $A_{j}$ и $A_{l}$ (т. е. $x_{0 j}=x_{0 l}$ ) проблема должна анализироваться совместно с достаточными условиями в каждом конкретном случае. При этом должны обязательно выполняться необходимые условия (35) и (36) по каждому из веществ $A_{j}$ и $A_{l}$. Проверка необходимых условий возникновения «мертвой зоны» одновременно по большему количеству веществ происходит аналогично.

Кроме того, возможна ситуация, когда «мертвая зона» по одному $\left(A_{j}\right)$ или одновременно по нескольким $\left(A_{j}, A_{l}\right.$ и т. д.) веществам уже возникла, но некоторые из отдельных реакций в схеме $(1)$ в центральной области $0 \leqslant x \leqslant x_{0 j}$ пористой гранулы продолжают идти. В этом случае возможность возникновения «мертвой зоны» по остальным веществам анализируется также с применением формул (35) и (36) с учетом того, что при этом вещества, по которым «мертвая зона» уже появилась, в реакциях не участвуют.

Перед применением условий (35) и (36) необходимо проанализировать уравнения математической модели (2) и (3) на наличие функциональных зависимостей между концентрациями $C_{i}$ отдельных веществ $A_{i}(i=1, \ldots, n)$. При их наличии они должны учитываться в формулах $(35)$ и (36). На это обратили внимание, в частности, авторы исследований $[21,22,25]$, применявшие необходимые условия возникновения «мертвой зоны», представленные в работе [3].

В качестве примера рассмотрим реакцию вида

$$
a_{1} A_{1}+a_{2} A_{2} \Rightarrow \text { Reaction Products, }
$$

протекающую со скоростью

$$
r_{1}=k C_{1}^{n} C_{2}^{m}
$$

в изотермических пористых гранулах катализатора шарообразной, цилиндрической или пластинчатой форм в стационарном режиме. Квазигомогенная модель (2) и (3) с учетом равенств (13) 
в этом случае примет вид:

$$
\begin{aligned}
& x^{-\alpha} \frac{d}{d x}\left(x^{\alpha} D_{1}^{*} \frac{d C_{1}}{d x}\right)=a_{1} g(x) r_{1}\left(C_{1}, C_{2}\right) \\
& x^{-\alpha} \frac{d}{d x}\left(x^{\alpha} D_{2}^{*} \frac{d C_{2}}{d x}\right)=a_{2} g(x) r_{1}\left(C_{1}, C_{2}\right) .
\end{aligned}
$$

Граничные условия (4) и (6) примут соответственно вид:

$$
\begin{gathered}
\left.D_{1}^{*} \frac{d C_{1}}{d x}\right|_{x=0}=0,\left.\quad D_{2}^{*} \frac{d C_{2}}{d x}\right|_{x=0}=0 ; \\
\left.D_{1}^{*} \frac{d C_{1}}{d x}\right|_{x=R_{S}}=\beta_{1}\left(C_{01}-C_{S 1}\right),\left.\quad D_{2}^{*} \frac{d C_{2}}{d x}\right|_{x=R_{S}}=\beta_{2}\left(C_{02}-C_{S 2}\right) .
\end{gathered}
$$

Умножим первое уравнение в системе $(42)$ на $a_{2}$, а второе - на $a_{1}$. Затем вычтем из первого второе уравнение и после преобразований с учетом граничных условий (43) и (44) получим:

$$
C_{2}=\frac{a_{2} D_{1}^{*}}{a_{1} D_{2}^{*}} C_{1}+C_{02}-\frac{a_{2} \beta_{1}}{a_{1} \beta_{2}} C_{01}-\frac{a_{2}}{a_{1}}\left(\frac{D_{1}^{*}}{D_{2}^{*}}-\frac{\beta_{1}}{\beta_{2}}\right) C_{S 1} .
$$

Применение формул (35) и (36) к кинетическому уравнению (41) приводит к следующим необходимым условиям возникновения «мертвой зоны»:

1. по веществу $A_{1}:|n|<1$;

2. по веществу $A_{2}:|m|<1$.

Из анализа уравнения (45) следует, что «мертвая зона» может образоваться одновременно по веществам $A_{1}$ и $A_{2}$, если справедливо равенство:

$$
C_{02}-\frac{a_{2} \beta_{1}}{a_{1} \beta_{2}} C_{01}=\frac{a_{2}}{a_{1}}\left(\frac{D_{1}^{*}}{D_{2}^{*}}-\frac{\beta_{1}}{\beta_{2}}\right) C_{S 1} .
$$

При этом формула (41) с учетом (45) и (46) примет вид:

$$
r_{1}=k\left(\frac{a_{2} D_{1}^{*}}{a_{1} D_{2}^{*}}\right)^{m} C_{1}^{n+m} .
$$

Применив к формуле (47) соотношения (35) и (36), получим необходимое условие появления «мертвой зоны» одновременно по веществам $A_{1}$ и $A_{2}$ :

$$
|n+m|<1
$$

5. Заключение. Проанализированы приближенные решения уравнений, описывающих массои теплоперенос в пористых гранулах катализатора шарообразной, цилиндрической и пластинчатой форм с учетом сопротивления внешнему массо- и теплообмену. Также обобщены необходимые условия существования «мертвой зоны» для произвольных каталитических процессов, протекающих на неизотермических пористых гранулах катализатора.

\section{СПИСОК ЛИТЕРАТУРЫ}

1. Андреев B. В. Анализ нестационарных режимов в пористых гранулах катализатора с гауссовым распределением активных центров по глубине// Вестн. Чуваш. ун-та. - 2003. - № 2. - С. 8-15.

2. Андреев B. В. «Мертвая зона» в пористой грануле катализатора для нестационарной параллельной реакции // Ж. физ. хим. - 2006. - 80, № 4. - С. 628-633.

3. Андреев B. В. Формирование «мертвой зоны» в пористых структурах в ходе процессов, протекающих в стационарном и нестационарном режимах// Обз. ж. хим. -2013 . - 3, № 3. - С. 262-293.

4. Андреев В. В. Исследование эффективности корочковых пористых гранул катализаторов// в кн.: Тр. Х Всеросс. конф. «Необратимые процессы в природе и технике». - М.: МГТУ им. Н. Э. Баумана, 2019. - C. 129-132.

5. Андреев В. В., Алексеев Б. В., Колъцов Н. И. «Мертвая зона» в пластинчатом зерне катализатора для сложных реакций со степенной кинетикой// Изв. вузов. Химия хим. техн. - 1992. - 35, № $11-12$. - C. $52-55$. 
6. Андреев В. В., Возяков В. И. Кольцов Н. И. Обтекание потоком реакционной смеси пористых гранул катализатора с химической реакцией на внутренней поверхности// Хим. физ. - 1994. — 13, № 11 . C. 55-64.

7. Андреев B. В., Грищай В. И. Моделирование неактивных зон в пористых гранулах катализатора и в биосенсоре// Мат. модел. - 2005. - 17, № 2. - С. 57-64.

8. Андреев B. В., Грицай В. И. «Мертвая зона» в пористых гранулах катализатора в нестационарном режиме при треугольной реакции// Изв. вузов. Химия хим. техн. - 2007. - 50, № 7. - С. 50-54.

9. Андреев В. В., Грицай В. И., Прилуцкий Ю. И. Исследование физико-химических параметров неактивных зон пористой гранулы катализатора// Докл. НАН Украины. - 2007. - 6. - С. 61-69.

10. Андреев В. В., Колъцов Н. И. «Мертвая зона» в пористых зернах катализатора для реакций с произвольной кинетикой // Докл. РАН. - 1993. - 332, № 5. - С. 581-584.

11. Андреев В. В., Кольцов Н. И., Возяков В. И. Условия возникновения «мертвой зоны» в зернах катализатора для реакций с произвольной кинетикой// Изв. вузов. Химия хим. техн. - 1993. - 36, № 11. - C. $61-66$.

12. Грицай В. И., Андреев В. В. Роль диффузии в формировании неактивных зон в пористых реакционнодиффузионных средах // Мат. модел. - 2006. - 18, № 12. - С. 88-94.

13. Колъцов Н. И., Андреев В. В. Об особенностях протекания простых и сложных реакций в пористых зернах катализатора// Кинетика и катализ. - 1995. - 36, № 1. - С. 77-81.

14. Andreev $V . V$. Conditions required to maximise the productivity of porous catalyst granules with a controlled activity profile// Mendeleev Commun. - 1998. - 2. - P. 77-79.

15. Andreev $V$. V. A mathematical treatment of the use of ultrasound in homogeneous and heterogeneous catalysis// Ultrason. Sonochem. - 1999. — 6, № 1-2. — P. 21-24.

16. Andreev $V$. V. A mathematical treatment of the use of ultrasound in a catalytic reactor with controlled activity profile// Proc. 15th Int. Symp. "Nonlinear Acoustics at the Turn of the Millenium" (Göttingen, Germany, 1-4 September 1999). — Melville-New York: Am. Inst. Phys., 2000. — P. 469-472.

17. Andreev V. V., Koltsov N. I. Optimal catalyst distribution on a non-isothermal porous granule for a monoirreversible reaction with arbitrary kinetics// Acta Chim. Sloven. - 1995. — 42, № 1. — P. 43-46.

18. Andreev V. V., Koltsov N. I., Ivanova A. F., Konstantinova N. V. On the possibility of increasing the effectiveness of a porous catalyst granule for a simple reaction in a non-stationary regime// Mendeleev Commun. - 1995. - 4. - P. 152-153.

19. Ellery A. J., Simpson M. J. An analytical method to solve a general class of nonlinear reactive transport models// Chem. Eng. J. — 2011. - 169, № 1-3. - P. 313-318.

20. Hernandez Aguirre A., Morales Cabrera M. A., Morales Zarate E., Rivera V. M., Puebla H., Hernandez Martinez E. Non-isothermal effectiveness factor for catalytic particles with non-Fickian diffusion// Int. J. Chem. Reactor Eng. — 2017. — 15, № 5 .

21. Król G., Szukiewicz M. Conditions of dead zone forming in porous catalyst pellets// Chem. Process Eng. - 2018. - 39, № 1. - P. 129-138.

22. Król G., Szukiewicz M., Chutkowski M. Formation of dead zone in catalytic particles in catalysis and biocatalysis — new alternative method of determination// Can. J. Chem. Eng. — 2019.

23. Satterfield C. N., Sherwood T. K. The Role of Diffusion in Catalysts. - London: Addison-Wesley, 1963.

24. Simpson M. J., Ellery A. J. An analytical solution for diffusion and nonlinear uptake of oxygen in a spherical cell// Appl. Math. Model. - 2012. — 36, № 7. - P. 3329-3334.

25. Szukiewicz M. K. Efficient numerical method for solution of boundary value problems with additional conditions// Brazil. J. Chem. Eng. — 2017. — 34, № 3. - P. 873-883.

26. York R. L., Bratlie K. M., Hile L. R., Jang L. K. Dead zones in porous catalysts: Concentration profiles and efficiency factors// Catal. Today. — 2011. - 160. — P. 204-212.

Андреев Всеволод Владимирович

Чувашский государственный университет имени И. Н. Ульянова, Чебоксары

E-mail: andreev_vsevolod@mail.ru 